\title{
Preloading based needle insertion with a concave probe to enhance targeting in breast tissue
}

\author{
Yo Kobayashi ${ }^{1 *}$, Maya Hatano ${ }^{1}$, Makiko Suzuki $^{1}$, Yasuyuki Shiraishi ${ }^{2}$, Tomoyuki Yambe ${ }^{2}$, Makoto Hashizume ${ }^{3}$ \\ and Masakatsu G Fujie ${ }^{1}$
}

\begin{abstract}
Target displacement cased by tissue deformation is a major technical challenge in ultrasound-guided needle insertion for breast tumor treatment or biopsy. We previously developed a preloading-based needle insertion method, which consists of a preloading phase and an insertion phase, and validated its placement accuracy. The present study furthered this research by focusing on a new concave preloading probe to enhance needle insertion targeting. With this probe, we evaluated the several probe size for different tumor diameters and locations to investigate the geometry effect on insertion accuracy. In this in vitro study, preloading-based needle insertion with concave probe of the same diameter as that of the tumor showed the highest placement accuracy for shallow tumors, while concave probe of a larger diameter than that of the tumor showed the highest placement accuracy for deep tumors. Overall, the concave preloading probe described in this study decreased needle placement error. Future research focused on its evaluation by structural analysis based on soft tissue modelling and deformation simulation.
\end{abstract}

Keywords: Needle insertion; Breast; Surgical robot; Preloading; Palpation

\section{Background}

Breast cancer accounts for more than 1 million of the estimated 10 million malignancies diagnosed worldwide each year [1]. Breast imaging technologies for early diagnosis of disease are improving along with treatments that may improve the prognosis [2]. There has been a recent trend toward minimally invasive procedures such as needle biopsy and radiofrequency ablation (RFA) therapy. In these procedures, ultrasound (US) imaging is widely used to guide insertion of a needle into tumor tissue because it has the advantages of real-time visualization and cost-effectiveness. During the procedure, a clinician holds an US probe with one hand and inserts the needle with the other. In the case of RFA therapy, the needle then emits radiofrequency energy from microwaves to heat and destroy the tumor lesion. Alternatively, the needle takes a sample of the tumor for

\footnotetext{
* Correspondence: you-k@fuji.waseda.jp

${ }^{1}$ Faculty of Science and Engineering (Research Institute for Science and Engineering), Waseda University, 59-309, 3-4-1 Okubo, 169-8555 Shinjuku-ku, Tokyo, Japan

Full list of author information is available at the end of the article
}

biopsy. For breast tumors, these therapies are promising as they are minimally invasive, cause less scarring, have quick recovery rates, and decrease cost.

\section{Problem}

However, technical challenges exist in needle insertion for breast tumor treatment. In spite of a real-time visible image from an US probe, accurate placement of the needle is still difficult, especially with small, early breast tumor lesions that require placement accuracy of only a few millimeters. Because the breast is soft, it can easily deform when a needle pierces it and advances toward an inner tumor. The deformation causes displacement of the tumor lesion. Thus, accurate needle insertion requires solving the problems of tissue deformation and target displacement.

\section{Related research}

Current research and development of robots and navigation systems for minimally invasive surgery is focused on improving needle insertion and placement [3-5]. For example, Bassan et al. reported a novel five-degrees-offreedom manipulator for percutaneous needle insertion 
[6]. A puncture-assisting robot operating under US guidance for use during procedures involving the prostate gland has also been developed [7]. External actuators have been used to manipulate the position of breast lesions $[8,9]$. In both studies, multiple actuators were used to decrease targeting error. In another study, a robotic medical needle insertion procedure using tissue manipulation was developed to improve targeting accuracy [10].

A number of research groups have also conducted studies to solve the problem of organ deformation associated with needle insertion [11]. For example, methods have been developed for mechanical modeling of breast deformations [12,13] and simulation of steerable needle insertion for prostate therapy [14-16]. Deformation of a planar tissue phantom during needle insertion has been investigated using a linear elastic material model $[17,18]$. Dehghan et al. determined the optimal angle of needle insertion and position using linear and nonlinear models [19]. Picinbono et al. described nonlinear and anisotropic elastic liver models for medical simulation [20]. A physics-based model for medical palpation of deformable tissue has also been reported [21]. Many research groups have used animal organs as models to investigate measurement details associated with needle insertion, puncture and friction force, and tissue stiffness (e.g., [22-24]).

\section{Motivation}

We have been researching the method to enhance the targeting in breast tissue, named a preloading-based needle insertion method. The concept of the method is that before inserting the needle, the breast is pressed by the preloading probe to stabilize the tumor as the needle approaches. During the needle insertion phase, the needle direction does not change to avoid damaging internal tissues. Insertion is stopped when the tumor position and needle tip are closest. Thus, this method has a preloading phase and a needle insertion phase to locate the preloading probe and pierce the breast tissue. The preloading phase hardens the tissue between the target and the needle tip to stabilize the tumor and make it easier to pierce the tissue during the needle insertion phase.

We previously reported this concept and its evaluation by numerical simulations and experiments both in vitro and in vivo. Smaller error with preloading-based insertion than with normal insertion was obtained in all experiments [25-28].

\section{Objectives and scope of this article}

The shape of preloading probe in our previous paper [25-28] was the round convex shape (termed 'convex probe') with a certain diameter. The shape and size of preloading probe is not limited to the condition and preloading concept can be extended to other shape and size of preloading probe. This paper focus on investigation to test the probe with round concave shape (termed 'concave' probe) and to evalauate preloading concept with concave probe to enhance accurate needle insertion (Figure 1). We assumed that the appropriate preloading probe diameter could be determined with respect to tumor diameter and location. More specifically, the appropriate diameter of the concave probe depends on the diameter of the tumor. In addition, the tumor location in the breast tissue might affect the determination of the appropriate probe diameter. The objective of the present paper was to evaluate these geometry effect of the preloading probe on insertion accuracy with respect to tumor diameter and location. In vitro and in vivo experiments were performed for this evaluation.

\section{Methods}

This section shows our evaluation of the preloading method with concave probe on needle insertion accuracy by in vitro experiments using a hog breast and in vivo experiments using a goat breast.

\section{Experimental setup of in vitro experiments}

Figure 2 depicts an overview of the in vitro experiments and the tip part of the needle insertion manipulator. Both normal needle insertion and preloading-based needle insertion with several concave probe diameters were conducted for comparison of insertion accuracy. In addition, all trials were performed with two tumor depths to reveal the geometry effect of the preloading probe with respect to the initial tumor location.

\section{Needle insertion manipulator}

The manipulator had four degrees of freedom for planar movements and components for positioning and integrating the apparatus. The positioning component had three serial joints with rotational degrees of freedom. The integrating component had two parts with separate drives: one for the preloading probe and the other for needle insertion. Preloading probes with different diameters could be alternately attached. Each preloading probe

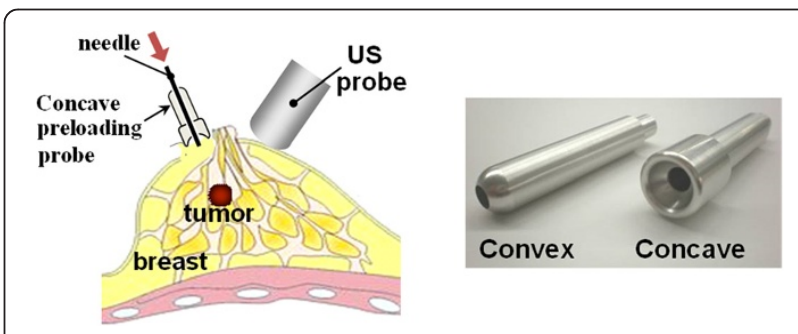

Figure 1 Conceptual sch. of preloading-based needle insertion with concave probe. The concept of the method is that before inserting the needle, the breast is pressed by the preloading probe to stabilize the tumor as the needle approaches. 

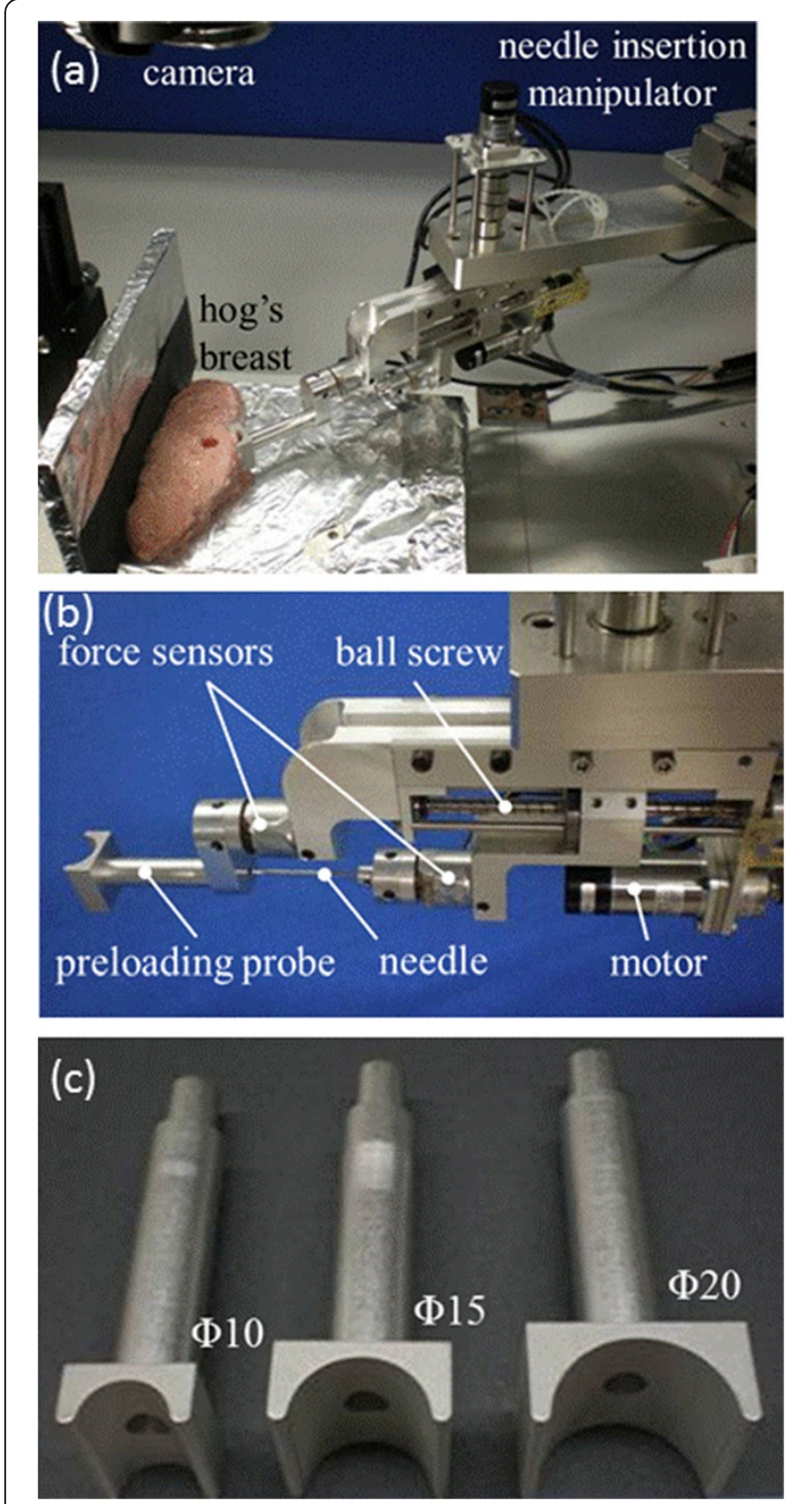

Figure 2 Experimental setup of in vitro experiment (needle insertion manipulator, camera, and hog breast). (a) Overview of in vitro experiment. (b) Enlarged view of the tip part of the needle insertion manipulator. (c) Concave probes in vitro experiment.

had a center hole for insertion of a needle of $2.0 \mathrm{~mm}$ diameter. Six-axis force/torque sensors (NANO 1.2/1, BL AUTOTEC) were attached to the root of the probe and needle to measure the pressing force and reaction force. We calculated needle tip and preloading probe tip positions from the angle of each joint of the manipulator, measured by encoders attached to the motors.

\section{Breast with mock tumor}

We used flattened hemispherical hog breast tissue, approximately $100 \mathrm{~mm}$ in diameter and $20 \mathrm{~mm}$ thick. A silicone cylinder, $10 \mathrm{~mm}$ in diameter and $20 \mathrm{~mm}$ long, served as a mock tumor embedded in the breast tissue.

\section{Tumor position recognition}

To operate the preloading probe and determine the needle placement accuracy, we tracked tumor location using a camera and an image-processing computer. We manually set the tumor lesion on the camera image, and the computer automatically tracked the lesion using the pattern-matching method.

\section{Tumor locations}

We compared needle placement accuracy at two tumor depths: 15 and $30 \mathrm{~mm}$. We regarded these depths as the distance between the top of the breast and a line $30^{\circ}$ from the perpendicular to the edge of the hemispherical breast tissue (Figure 3). We selected these two depths to be as far as possible yet within the operating restriction of RFA to maintain distance from the skin and costae. Both normal insertion and preloading-based needle insertion were conducted at these two tumor depths.
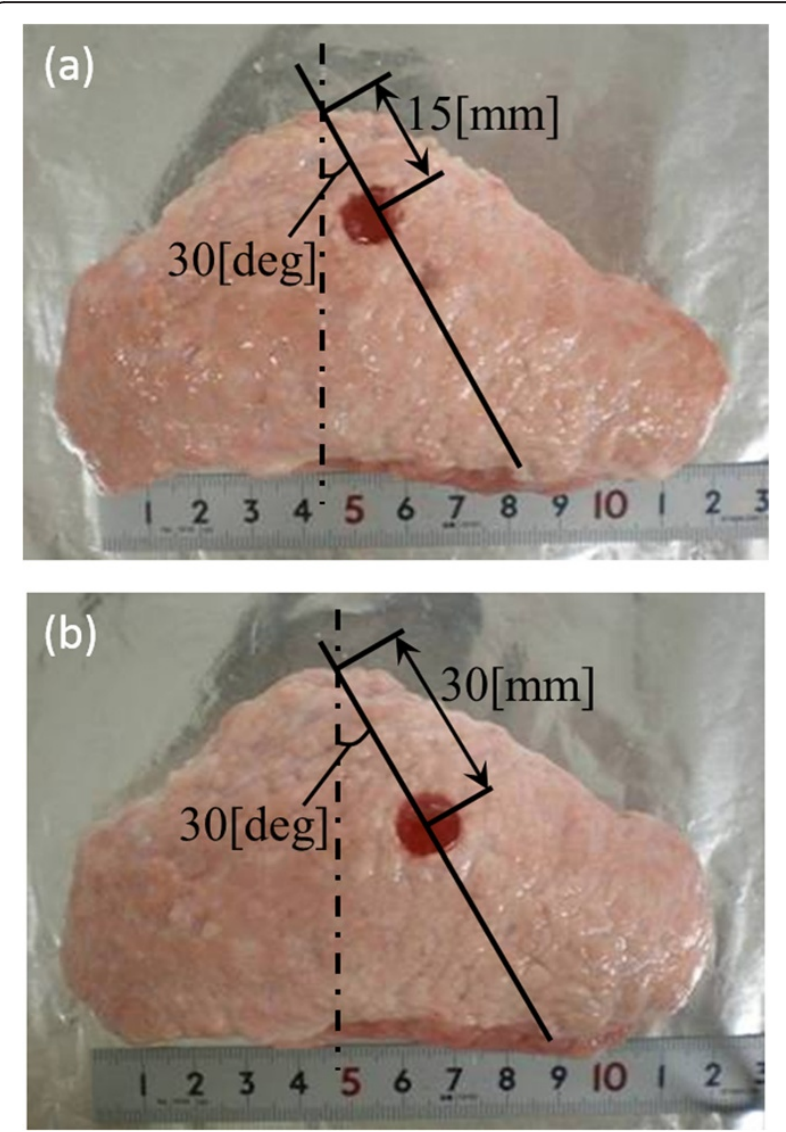

Figure 3 Hog breast with cylindrical mock tumor made of silicone. (a) Shallow tumor location. (b) Deep tumor location. 


\section{Insertion path}

For cosmetic reasons, the superior edge of the areola is a preferred location for initial insertion. Consequently, the top of the breast was selected as the initial insertion point for the experiments. The direction of needle insertion was downward from the upper half of the breast to the center of the tumor.

\section{Condition of normal insertion}

The needle was advanced at a constant velocity of $4 \mathrm{~mm} / \mathrm{s}$ toward the center of the initial position of the tumor lesion. Insertion was complete when the tumor position and needle tip were closest. We measured the force loaded on the needle, the tumor position, and the needle tip position to determine the placement error. Four trial insertions were performed on four hog breast samples at two different tumor depths (total of 32 trials).

\section{Condition of preloading-based needle insertion}

Preloading-based needle insertion was conducted with three preloading probe diameters $(10,15$, and $20 \mathrm{~mm}$ ) with a concave shape. They were alternately attached during the experiments to compare the geometry effect of the preloading probe (Figure 2c). During the preloading phase, the preloading probe pressed the hog breast toward the tumor lesion. The probe pressed the breast at $4 \mathrm{~mm} / \mathrm{s}$ as the probe axis was adjusted to the center of the tumor lesion using the camera information of tumor position. We stopped the preloading when the reaction force reached $2.0 \mathrm{~N}$. We previously compared insertion accuracy with different loading forces and suggested $2.0 \mathrm{~N}$ as the appropriate pressing force that would enhance robustness in insertion accuracy [28]. After the preloading phase, we moved to the needle insertion phase. We inserted the needle at a constant velocity of $4 \mathrm{~mm} / \mathrm{s}$. During the needle insertion phase, the needle direction did not change. Insertion stopped when the tumor position and needle tip were closest. Again, we measured the force loaded on the needle, the tumor position, and the needle tip position to determine the placement error. For each of the three probes of different diameters, four trial insertions were performed on four hog breast samples at two different tumor depths (total of 96 trials). They were performed in random order to avoid skewing the results.

\section{Needle placement error}

We stopped the needle movement when the needle tip reached the intersection of the needle axis and the vertical axis and went through the target position. Accurate insertion requires alignment of the needle axis with the target lesion at the end of insertion. Insertion accuracy is evaluated as the placement error, L, which is defined by the distance between the needle axis and the center of the mock tumor at the end of the needle insertion:

$$
L=\frac{\left|a X_{c}+b Y_{c}+c\right|}{\sqrt{a^{2}+b^{2}}}
$$

where $(\mathrm{Xc}, \mathrm{Yc})$ are the coordinates of the center of the tumor lesion. The axis direction of the insertion is determined as follows:

$$
a x+b y+c=0
$$

\section{Experimental setup of in vivo experiment}

We also evaluated the accuracy of preloading-based needle insertion in vivo. A goat breast was used as the sample because its stiffness is similar to that of the human breast and because the size of goats allows for relatively easy maneuvering compared with heavy animals such as porcine. We compared preloading-based insertion with both concave probe and normal insertion.

All animals received humane care in accordance with "Guide for the Care and Use of Laboratory Animals" published by the National Institute of Health (NIH publication 85-23, revised 1985), "Guidelines for Proper Conduct of Animal Experiments" formulated by Science Council of Japan (2006), and the guidelines determined by the Institutional Animal Care and Use Committee of Tohoku University.

\section{Needle insertion manipulator}

Figure 4 depicts the needle insertion manipulator and the US equipment used for in vivo experiments. We previously developed an US-guided needle insertion manipulator [29], and parts of this manipulator were used in this experiment to set the needle orientation. The manipulator has two degrees of freedom to determine the path for inserting the needle. In addition, the insertion component for the insertion method was designed to have two parts with separate drives; one for the preloading probe and the other for needle insertion. The preloading probe had a $10-\mathrm{mm}$ diameter with a center hole for inserting a $1.5-\mathrm{mm}$-diameter needle. We prepared a preloading probe with a round concave shape with a

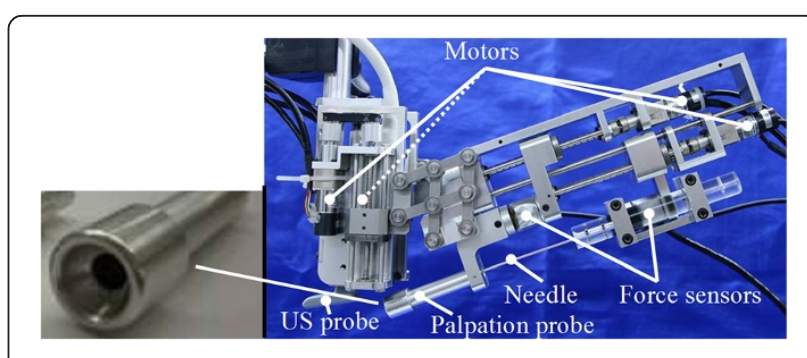

Figure 4 Needle insertion manipulator for in vivo experiments. 
diameter of 10-mm. Six-axis force/torque sensors (NANO 1.2/1, BL AUTOTEC) were attached to the root of the probe and needle.

\section{Goat breast with mock tumor}

The goat was maintained under general anesthesia. A silicone sphere (10 mm diameter) covered with a blood vessel prosthesis was used to prevent movement of the breast tissue (Figure 5). A mock tumor lesion was embedded into the breast $15 \mathrm{~mm}$ under the skin. Embedding of the mock tumor was performed on the day prior to experimentation via an incision in the breast made using an electrosurgical knife. The incision was then sutured and closed. The conditions were identical to those of the in vitro experiment (i.e., tumor size, $10 \mathrm{~mm}$; tumor depth, $15 \mathrm{~mm}$; and probe diameter, $10 \mathrm{~mm}$ ), which resulted in high insertion accuracy (ref. section Experiment with shallow tumor location and section Preloading-based needle insertion with concave probe).

\section{Experimental setup}

Figure 5 shows the experimental setup of in vivo experiments. First, the surgeon searched the target inferior mock cancer by manipulating the US probe attached to the needle insertion manipulator. Next, the surgeon fixed the probe with the needle insertion manipulator using the endoscope holding device at the target location to visualize the target inferior mock cancer on the US image. Finally, the surgeon used the Graphical User Interface (GUI) to order the needle insertion path from the computer.

\section{Target position measurement}

To determine needle placement accuracy, we measured the target location using US equipment and an imageprocessing computer.

\section{Needle and preloading probe position}

We calculated the tip positions of the needle and the palpation probe from the angle of each joint of the manipulator, as measured by encoders attached to the motors.

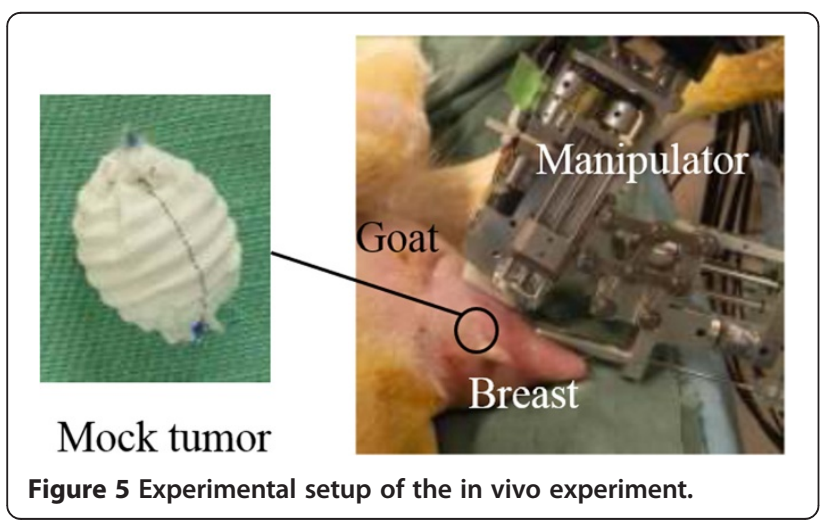

\section{Needle insertion error}

The definition of insertion error was identical to that for the in vitro experiments.

\section{Condition of normal insertion}

We detected the target lesion using the US image and fixed the manipulator to place the lesion on the US image screen. The needle direction was straight downward from near the areola at a constant velocity of $4 \mathrm{~mm} / \mathrm{s}$ toward the center of the initial position of the target lesion. Insertion was complete when the target position and needle tip were closest. We measured the force loaded on the needle, target position, and needle tip position to determine the placement error. We performed three trial experiments.

\section{Condition of preloading-based insertion}

The preloading probe pressed near the areola of the goat breast. The probe moved at $4 \mathrm{~mm} / \mathrm{s}$ during the preloading phase with its position directed at the center of the target lesion on the probe axis. The target lesion was measured from the US image using the patternmatching method. The preloading phase was finished when the force loaded on the preloading probe reached $0.75 \mathrm{~N}$. A needle was then inserted at a constant velocity of $4 \mathrm{~mm} / \mathrm{s}$. Insertion stopped when the target position and needle tip were closest. We measured the force loaded on the needle, target position, and needle tip position to determine the insertion error. We performed three trial experiments.

\section{Results}

\section{In vitro experiment}

\section{Experiment with shallow tumor location}

Figure 6 shows the initial condition of the hog breast tissue and deformation after needle insertion under four different insertion conditions (normal needle insertion and preloading-based needle insertion with three probe diameters). The tumor was greatly displaced under normal needle insertion, and preloading enhanced the placement accuracy. Figure 7 shows the insertion errors under all preloading probe conditions. In Figure 7, "0" represents the results of normal insertion. The average placement error with normal needle insertion was $3.4 \mathrm{~mm}$. Preloading-based needle insertion with any preloading probe diameter reduced the placement error compared with normal insertion. Preloading-based insertion with the $10-\mathrm{mm}$ probe (the same diameter as that of the tumor) reduced the placement error the most. The average placement error was $0.50 \mathrm{~mm}$, which was approximately $15 \%$ of the error with normal insertion. Figure 7 also shows a tendency for reduced placement error with smaller preloading probe diameters. We conducted statistical analyses to evaluate these result. We 


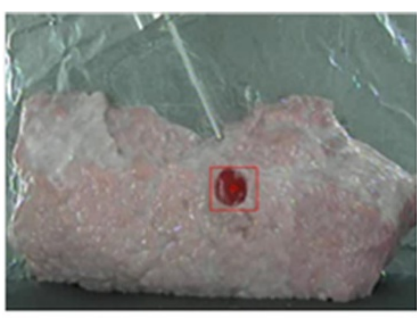

(a-1) Initial condition (Normal)

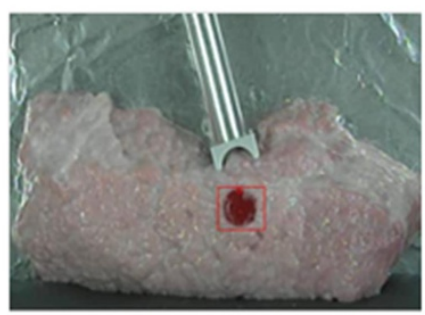

(b-1) Initial condition (Probe 10)

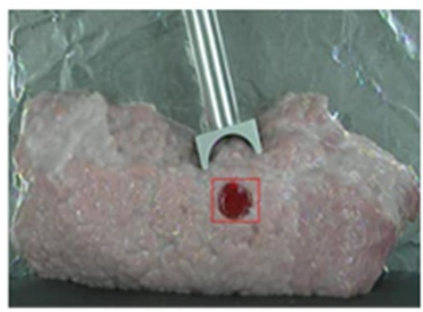

(c-1) Initial condition (Probe 15)

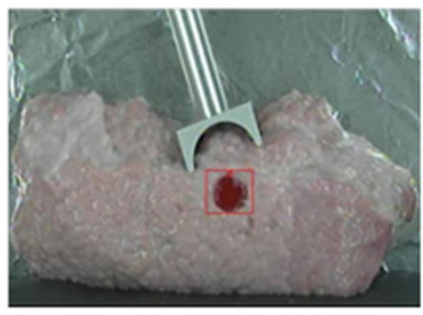

(d-1) Initial condition (Probe 20)

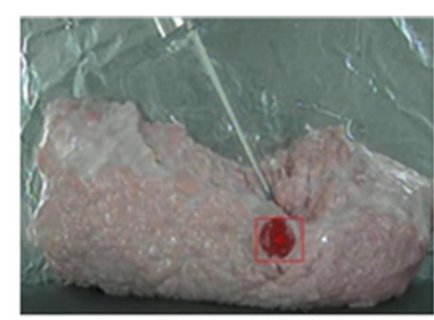

(a-2) Final condition (Normal)

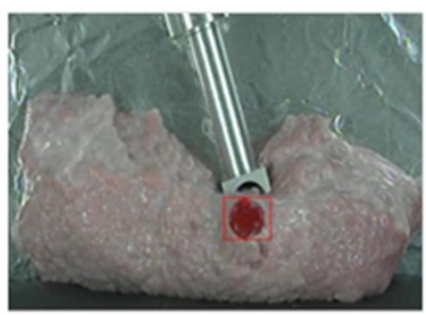

(b-2) Final condition (Probe 10)

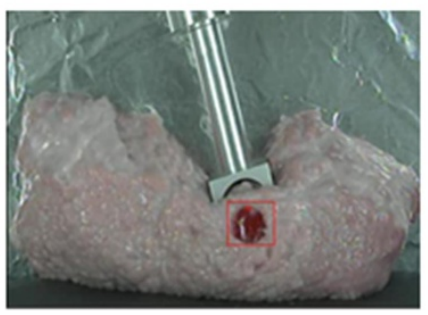

(c-2) Final condition (Probe 15)

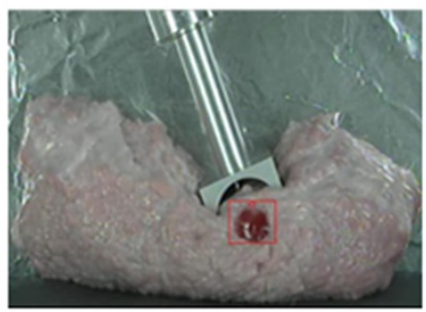

(d-2) Final condition (Probe 20)

Figure 6 Camera image of preloading-based needle insertion for shallow tumor.

performed post hoc multiple comparisons (Steel-Dwass nonparametric method) to determine which preloading probe elicited the improvement in insertion. Table 1 show the results of the multiple comparisons analyses. There were significant differences $(\mathrm{p}<0.05)$ between normal insertion and preloading method with 10 and $15 \mathrm{~mm}$ diameter.

\section{Experiment with deep tumor location}

Figure 8 shows the initial condition of the hog breast tissue and deformation after needle insertion under four different insertion conditions (normal needle insertion and preloading-based needle insertion with three probe diameters). Figure 9 shows the insertion errors under all preloading probe conditions. In Figure 9, "0" represents the results of normal insertion. The average placement error of normal needle insertion was $5.6 \mathrm{~mm}$. Preloading-based needle insertion with any preloading probe diameter reduced the placement error. The average placement error with 10-, 15-, and 20-mm-diameter probes were $2.6,2.2$, and $1.6 \mathrm{~mm}$, respectively. They resulted in approximately $46 \%, 39 \%$, and $29 \%$ of the error obtained with normal needle insertion. These results suggest a tendency for reduced placement error with larger preloading probes. The highest accuracy was achieved not by the probe with the same diameter as 


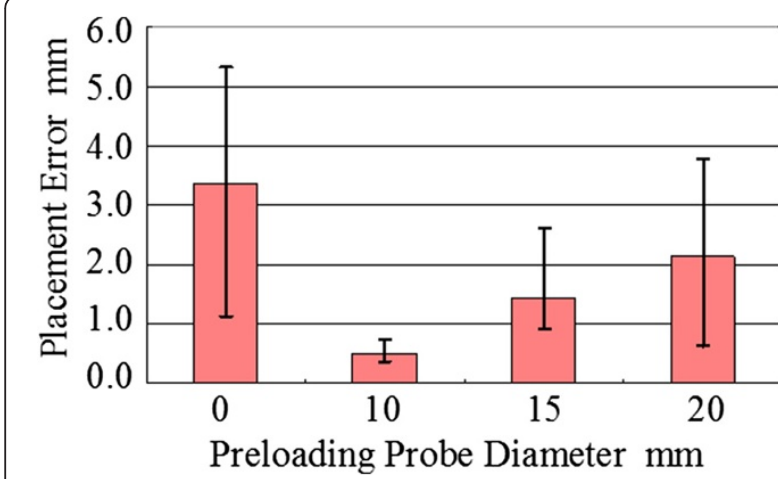

Figure 7 Insertion errors for each experimental condition with shallow tumor location. Normal insertion has a preloading probe diameter of $0 \mathrm{~mm}$.

that of the tumor, but by the probe with twice the diameter of that of the tumor. We conducted statistical analyses to evaluate these result. The procedures were same as that of shallow tumor and Table 2 show the results. There were significant differences $(\mathrm{p}<0.05)$ between normal insertion and preloading method with $20 \mathrm{~mm}$ diameter.

\section{In vivo experiment}

\section{US image during insertion}

Figure 10 shows an example of the US image during the experiment. The image allowed for successful location of the target position.

\section{Comparison with each insertion}

Figure 11 shows the relationship between the shape of the probe and the insertion error (average data of three experiments). In Figure 11, "None" represents the results of normal insertion. The error $\mathrm{L}$ for normal insertion was approximately $1.7 \mathrm{~mm}$. By contrast, the error was $1.0 \mathrm{~mm}$ for the preloading method with the concave probe. These data suggest that the error in needle displacement for preloading-based insertion with concave probe is significantly less than that of normal insertion.

Table 1 Results of statistical tests for insertion errors among different probe diameter in shallow tumor experiment

\begin{tabular}{lllll}
\hline Probe size & $\mathbf{0}$ & $\mathbf{1 0}$ & $\mathbf{1 5}$ & $\mathbf{2 0}$ \\
\hline 0 & & - & - & - \\
10 & $0.00006^{* *}$ & & - & - \\
15 & $0.01^{* *}$ & $0.02^{* *}$ & & - \\
20 & $0.2^{\text {n.s. }}$ & $0.008^{* *}$ & $0.7^{\text {n.s. }}$ & \\
\hline
\end{tabular}

P-value $<0.10$ are *. P-value $<0.05$ are **. n.s, not significant.

\section{Discussion}

Preloading-based needle insertion with concave probe Preloading-based needle insertion with all concave probe diameters showed better placement accuracy than did normal needle insertion. This can be said under conditions of both short and long distances between the tumor and the initial insertion point. Our data from the in vivo experiment also suggests that pressing by the concave probe reduces needle placement error. The major reason to enhance needle insertion accuravy is same as the condition with convex shape probe [28] and described as follows: Many related works have stated that the main puncture event is designated by a peak force applied to the needle that is preceded by a steady rise followed by a sharp decrease [22,23]. That is, when a needle punctures the breast, it does not immediately cut the tissue; instead, it first pushes the tissue and then punctures it. During this phase, tumor displacement is increased. The preloading method enhanced the needle insertion accuracy because the tumor displacement during needle insertion is decreasing. Our previous report also suggested that breast tissue with high strain displays nonlinear characteristics and higher stiffness parameters [28]. Pressing by the preloading probe results in high strain in the tissue surrounding the tumor. This spreads the tissue to not only stabilize it, but also to produce a rapid increase in stress near the needle. Puncture thus occurs with little tumor displacement. The concave probe is especially supposed to harden the tissue surrounding the tumor as discussed in this section later. The other potential advantage of concave shape stabilize the tumor at right angles to the direction of needle insertion. The futher investigation should be carried out about this issue while the advantage was partially supported by our previous result [27].

\section{Geometry effect of preloading probe}

For shallow tumors $(15 \mathrm{~mm})$, preloading-based needle insertion with the probe of the same diameter as that of the tumor showed the highest placement accuracy. In contrast, for deep tumors $(30 \mathrm{~mm})$, insertion with larger-diameter probes showed higher placement accuracy. It is supposed that a probe with the same diameter as that of the tumor would stabilize shallow tumors most effectively. The probe with the same diameter as that of the tumor could make high-stiffness elements surrounding the tumor to firmly stabilize it (Figure 12a) in shallow tumor situation. However, there might be a gap between the stiffened tissue and the tumor during insertion with larger-diameter probes (Figure 12b). This gap allows for tumor displacement, resulting in lower accuracy. We also obtained a contrasting result for deep tumors. The largest probe, twice the diameter of the tumor, achieved the highest accuracy for deep tumors. 


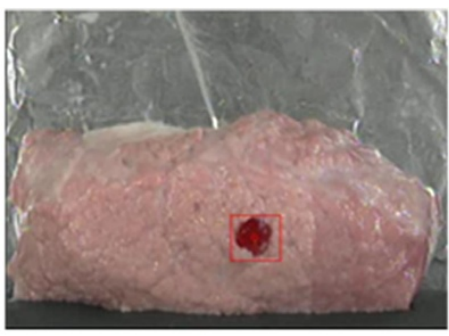

(a-1) Initial condition (Normal)

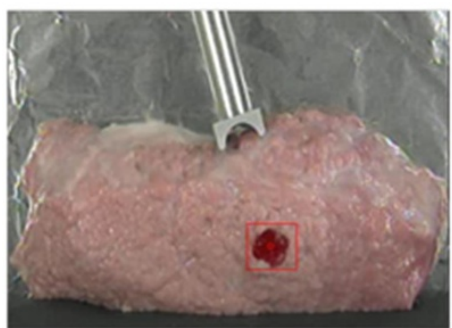

(b-1) Initial condition (Probe 10)

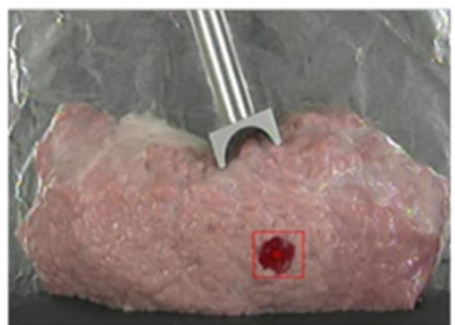

(c-1) Initial condition (Probe 15)

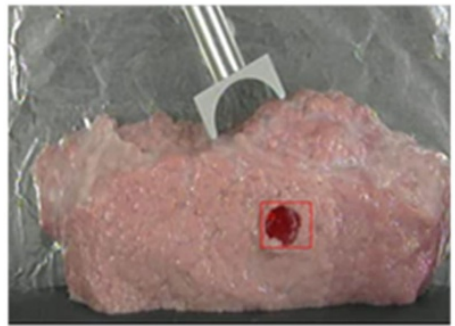

(d-1) Initial condition (Probe 20)

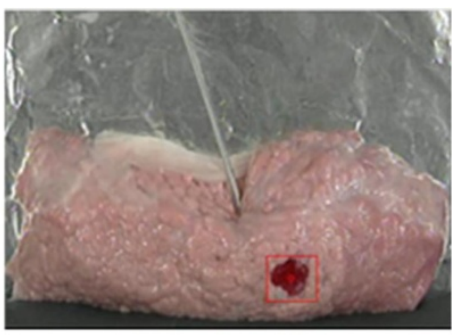

(a-2) Final condition (Normal)

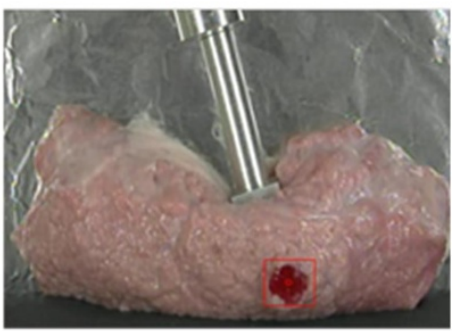

(b-2) Final condition (Probe 10)

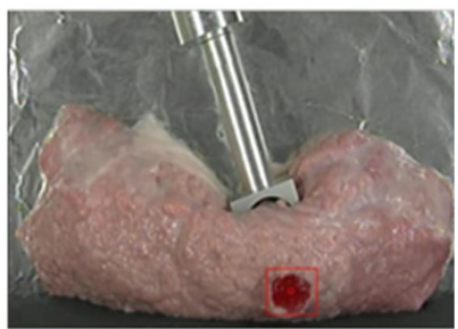

(c-2) Final condition (Probe 15)

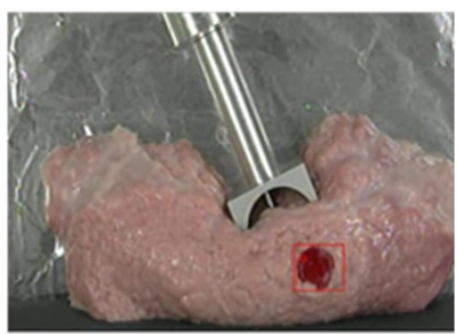

(d-2) Final condition (Probe 20)

Figure 8 Camera image of preloading-based needle insertion for deep tumor.

While the preloading probe approached the tumor and half-surrounded it under shallow conditions in the experimental result (Figure 6), there was still distance between the preloading probe and the tumor at the end under deep conditions (Figure 8). For deep tumors, only the tissue above the tumor might have been stiffened, and the largest probe make the largest stiffened area above the tumor to enhance accuracy (Figure 12c and d). It is supposed from these results that concave-shaped preloading probe with appropreciate size make the situation spreadding the high-stiffness tissue around the tumor effectively. It is also supported that the appropriate diameter of the concave probe depends on the diameter of the tumor. In addition, the tumor location in the breast tissue might affect the determination of the appropriate probe diameter.

\section{Advantage of preloading-based needle insertion}

preloading method has following advantages: (1) The success rate is high because tumor displacement is small. (2) Tissue damage is minimal because the insertion depth is smaller and the needle line is straight, not 


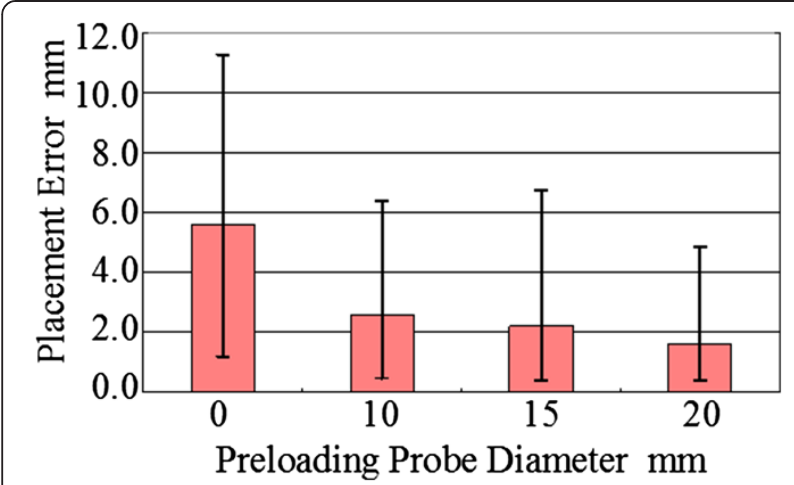

Figure 9 Average errors for each experimental condition with deep tumor location. Normal insertion has a preloading probe diameter of $0 \mathrm{~mm}$.

steered through the tissue. (3) The procedure is fast because the same device sequentially presses and inserts the needle. Furthermore, compared with the tissue manipulation method [8] and [9], the system has potential to be small and cost-effective because few actuators are needed. These advantages are same between the probe with convex and cocave shape. Meanwhile, we have advocated preloading efficiency with concave probe to minimize tumor displacement during needle insertion. The concave probe is expected to harden the tissue surrounding the tumor more effectively and stabilize it at right angles to the direction of needle insertion, resulting in effective reduction of tumor displacement. Further investigation is required to clearly discuss the comparison of convex and concave probe in preloading method.

\section{Simulation analysis}

Major limitation of this study is about structural analysis based on soft tissue modelling. As shown in our previous research [28], the simulation analysis may reveals the several situation and condition discussed above. We have not carried out simulation analysis because our simulation does not include the appropreciate collision detection algorism between the probe and breat tissue, which is one of the most difficult problem in soft tissue

Table 2 Results of statistical tests for insertion errors among different probe diameter in deep tumor experiment

\begin{tabular}{lllll}
\hline Probe size & $\mathbf{0}$ & $\mathbf{1 0}$ & $\mathbf{1 5}$ & $\mathbf{2 0}$ \\
\hline 0 & & - & - & - \\
10 & $0.03^{\text {n.s. }}$ & & - & - \\
15 & $0.07^{*}$ & $0.07^{\text {n.s. }}$ & & - \\
20 & $0.004^{* *}$ & $0.4^{\text {n.s. }}$ & $0.984^{\text {n.s. }}$ & \\
\hline
\end{tabular}

P-value $<0.10$ are *. P-value $<0.05$ are **. n.s, not significant.

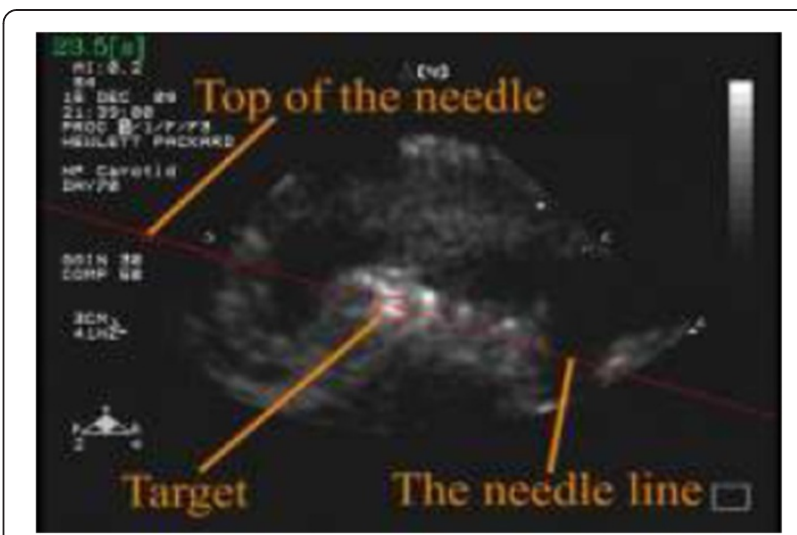

Figure 10 Representative US image during experiment.

modelling and simulation. Future research will focus on clarification of the tendency for a geometry effect of the preloading probe on placement accuracy with respect to tumor size and location based on simulation analysis. Moreover, the probe size can be optimized with respect to tumor size and location via repeated simulation anlysis with several probe size. The revealed tendency would also have the potential to suggest the new approach that a probe diameter changes in real time.

\section{Boundary condition}

The simplified experimental condition of the breast (i.e.. a half-cylinder shape with a single tissue type and simplified boundary condition) is one of the limitations of the present work. We plan to develop a system with heterogeneous patient-specific modeling, which incorporates features such as shape, regions of each tissue and boundary conditions, using preoperative CT/MRI

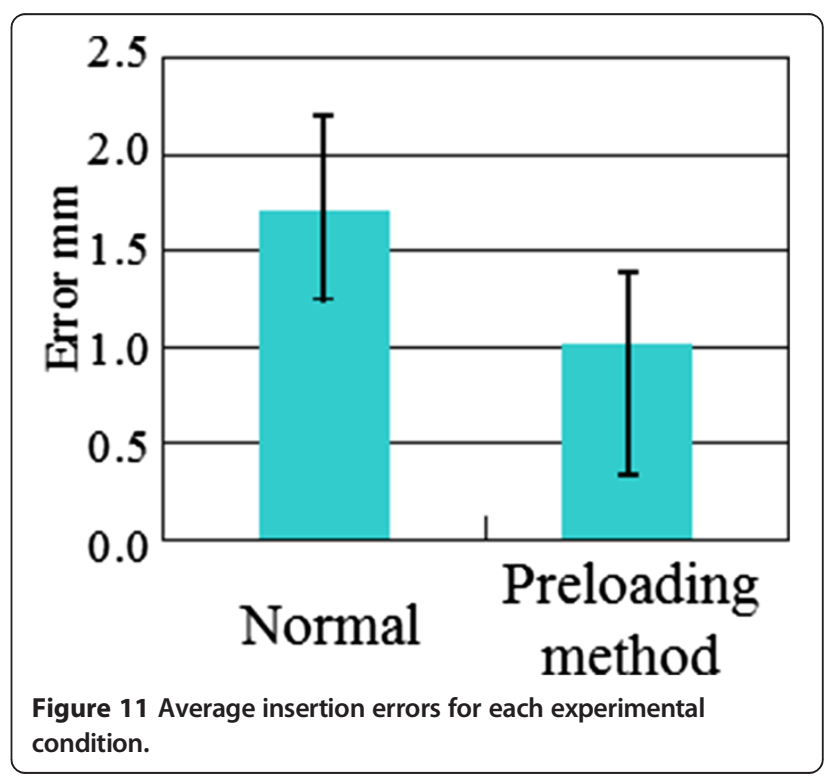


(a)

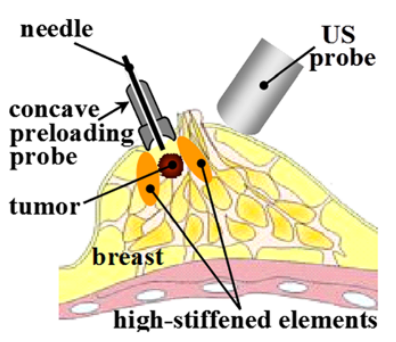

(b)

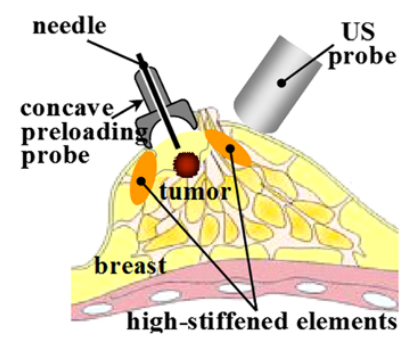

Shallow tumor location (c)

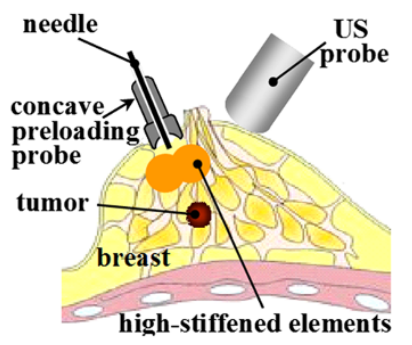

(d)

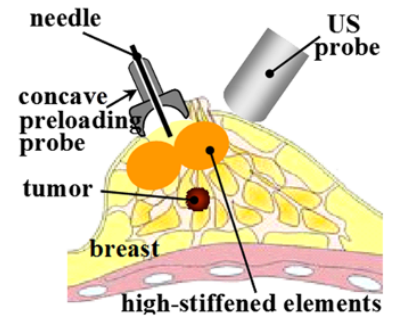

Deep tumor location

Figure 12 Conceptual scheme of Preloading method. (a) same diameterr probe as shallow tumor. (b) Larger diameter probe than shallow tumor. (c) same diameter as deep tumor. (d) larger diameter than deep tumor.

diagnostic images. Moreover, the collision between ultrasound probe and breast should be included in these boundary condition setteing.

\section{Conclusions}

We have described a needle insertion method involving tissue preloading to provide accurate needle insertion. The mechanical preloading probe locates tumor tissue and reduces tissue displacement during needle insertion by pressing the breast tissue. This paper focuses on investigation to evalauate preloading concept with concave probe to enhance accurate needle insertion. We compared preloading-based needle insertion using three concave probe diameters among one another and with normal insertion by experiments in vitro under conditions of two different tumor depths. Preloading-based needle insertion with a probe of the same diameter as that of the tumor showed the highest placement accuracy for the shallow tumor location. On the other hand, preloading-based insertion with a probe of larger diameter than that of the tumor showed higher placement accuracy for the deep tumor location. These results assumed that the appropriate preloading probe diameter could be determined with respect to tumor diameter and location. Our data from the in vivo experiment also suggest that pressing by the concave probe reduces needle placement error.

We will also research the integration of the palpation system into our system. A preloading probe will be used for both palpation and preloading. Reaction force applied to the preloading probe will indicate the tumor direction.
Our goal is to construct a surgical robot that incorporates both the needle insertion and palpation systems for clinical application.

\section{Competing interest}

The authors have no potential competing interest.

\section{Authors' contributions}

YK devised the basic concept of the overall system, technically constructed the system and drafted the manuscript. MH and MS technically constructed the system, carried out the experiments and helped to draft the manuscript. YS and TY devised the research plan of in vivo experimental based on the clinical point of view and gave the clinical discussion of the results. $\mathrm{MH}$ devised the basic concept and drew the research design based on the clinical point of view. MF devised the basic concept and drew the research design of the overall study. All authors read and approved the final manuscript.

\section{Acknowledgments}

This work was supported in part by the Global Centers of Excellence Program "Global Robot Academia," Waseda University, Tokyo, Japan; HighTech Research Center Project from the Ministry of Education, Culture, Sports, Science and Technology; a Grant-in-Aid for Scientific Research (A) (23240081); and a Grant-in-Aid for Young Scientists (B) (23700585).

\section{Author details}

${ }^{1}$ Faculty of Science and Engineering (Research Institute for Science and Engineering), Waseda University, 59-309, 3-4-1 Okubo, 169-8555 Shinjuku-ku, Tokyo, Japan. ${ }^{2}$ Department of Medical Engineering and Cardiology, Institute of Development, Aging and Cancer, Tohoku University, Sendai, Japan.

${ }^{3}$ Faculty of Medical Sciences, Kyushu University, Fukuoka, Japan.

Received: 26 December 2013 Accepted: 7 October 2014 Published online: 20 October 2014

\section{References}

1. Ferlay J, Bray F, Pisani P, Parkin DM (2001) GLOBOCAN 2000: cancer incidence, mortality and prevalence worldwide. IARC CancerBase :5

2. Vlastosa G (2007) Minimally invasive approaches for diagnosis and treatment of early-stage breast cancer. Oncologist 12:1-10 
3. Kazanzides P, Fichtinger G, Hager GD, Okamura AM, Whitcomb LL, Taylor RH (2008) Surgical and interventional robotics. IEEE Robotics \& Automation Magazine 15:122-30

4. Fichtinger G, Kazanzides P, Okamura AM, Hager GD, Whitcomb LL, Taylor RH (2008) Surgical and interventional robotics: part II. IEEE Robotics \& Automation Magazine 15:94-102

5. Hashizume M, Yasunaga T, Tanoue K, leiri S, Konishi K, Kishi K, Nakamoto H, Ikeda D, Sakuma I, Fujie M, Dohi T (2008) New real-time MR image-guided surgical robotic system for minimally invasive precision surgery. Int J Comp Ass Rad Surg 2:317-25

6. Bassan HS, Patel RV, Moallem M (2009) Novel manipulator for percutaneous needle insertion: design and experimentation. IEEE/ASME Trans Mechatron 14:746-61

7. Fichtinger G, Fiene JP, Kennedy CW, Kronreif G, lordachita I, Song DY, Burdette EC, Kazanzides P (2008) Robotic assistance for ultrasound-guided prostate brachytherapy. Med Image Anal 12:535-45

8. Vishnu G, Sarkar N, Podder TK (2008) Robot assisted real-time manipulation for breast biopsy. IEEE Proc 2008 Int Conf Robot Automation :2515-2515

9. Mallapragada VG, Sarkar N, Podder TK (2009) Robot-assisted real-time tumor manipulation for breast biopsy. IEEE Trans Robot 25:316-24

10. Torabi M, Hauser K, Alterovitz R, Duindam V, Goldberg K (2009) Guiding medical needles using single-point tissue manipulation. Proc 2009 IEEE Int Conf Robot Automation :2705-10

11. Larson BT, Erdman AG, Tsekos NV, Yacoub E, Tsekos PV, Koutlas IG (2004) Design of an MRI-compatible robotic stereotactile device for minimally invasive interventions in the breast. J Biomech Eng 126:458-65

12. Azar FS, Metaxas DN, Schnall MD (2001) A deformable finite element model of the breast for predicting mechanical deformations under external perturbations. Acad Radiol 8:965-75

13. Azar FS, Metaxas DN, Schnall MD (2002) Methods for modeling and predicting mechanical deformations of the breast under external perturbations. Med Image Anal 6:1-27

14. Alterovitz R, Goldberg K, Okamura A (2005) Planning for steerable bevel-tip needle insertion through 2D soft tissue with obstacles. Proc 2005 IEEE Int Conf Robot Automation :1652-7

15. Alterovitz R, Goldberg KY, Pouliot J, Hsu IC (2009) Sensorless motion planning for medical needle insertion in deformable tissues. IEEE Trans Inf Technol Biomed 13:217-25

16. Chentanez N, Alterovitz R, Ritchie D, Cho J, Hauser K, Goldberg K, Shewchuk JR, O'Brien JF (2009) Interactive simulation of surgical needle insertion and steering. ACM Trans Graph 88:1-10

17. DiMaio SP, Salcudean SE (2003) Needle insertion modelling and simulation. IEEE Trans Robot Automation 19:864-75

18. DiMaio SP, Salcudean SE (2005) Interactive simulation of needle insertion model. IEEE Trans Biomed Eng 52:1167-79

19. Dehghan E, Salcudean SE (2007) Needle insertion point and orientation optimization in non-linear tissue with application to brachytherapy. Proc 2007 IEEE Int Conf Robot Automation :2267-72

20. Picinbono G, Delingette $H$, Ayache N (2001) Nonlinear and anisotropic elastic soft tissue models for medical simulation. Proc 2001 IEEE Int Conf Robot Automation :1370-5

21. Daniulaitis V, Alhalabi MO, Kawasaki H, Tanaka Y, Hori T (2004) Medical palpation of deformable tissue using physics-based model for haptic interface robot. Proc 2004 Int Conf Intell Robots Systems :3907-11

22. Okamura AM, Simone C, O'Leary MD (2004) Force modeling for needle insertion into soft tissue. IEEE Trans Biomed Eng 51:1707-16

23. Heverly M, Dupont P, Triedman J (2005) Trajectory optimization for dynamic needle insertion. Proc 2005 IEEE Int Conf Robot Automation :1646-51

24. Mahvash $M$, Dupont $P$ (2009) Fast needle insertion to minimize tissue deformation and damage. Proc 2005 IEEE Int Conf Robot Automation :3097-102

25. Kobayashi Y, Suzuki M, Konishi K, Hashizume M, Fujie MG (2008) Development of a novel approach, 'palpation based needle insertion,' for breast cancer treatment. Proc 2008 IEEE Int Conf Robotics Biomimetics :505-11

26. Kobayashi Y, Suzuki M, Kato A, Konishi K, Hashizume M, Fujie MG (2009) A robotic palpation-based needle insertion method for diagnostic biopsy and treatment of breast cancer. Proc 2009 IEEE Int Conf Intell Robots Systems :5534-9
27. Hatano M, Kobayashi Y, Suzuki M, Shiraishi Y, Yambe T, Konishi K, Hashizume M, Fujie MG (2011) In vitro and in vivo validation of robotic palpation-based needle insertion method for breast tumor treatment. Proc 2011 IEEE Int Conf Robot Automation :392-7

28. Kobayashi Y, Suzuki M, Kato A, Hatano M, Konishi K, Hashizume M, Fujie MG (2012) Enhanced targeting in breast tissue using a robotic tissue preloading-based needle insertion system. IEEE Trans Robot 28(3):710-722

29. Kobayashi Y, Hong J, Hamano R, Okada K, Fujie MG, Hashizume M (2011) Development of a needle insertion manipulator for support during central venous catheterization. Int J Med Robot Comp Assist Surg 8(1):34-44

\section{doi:10.1186/s40648-014-0017-4}

Cite this article as: Kobayashi et al:: Preloading based needle insertion with a concave probe to enhance targeting in breast tissue. $R O B O M E C H$ Journal 2014 1:17.

\section{Submit your manuscript to a SpringerOpen ${ }^{\odot}$ journal and benefit from:}

- Convenient online submission

- Rigorous peer review

- Immediate publication on acceptance

- Open access: articles freely available online

- High visibility within the field

- Retaining the copyright to your article

Submit your next manuscript at $>$ springeropen.com 